\title{
Efficient Analysis of Inhomogeneous Dielectric Waveguides with Dielectric Strips and Microstrips Using Discrete Mode Matching Method
}

\author{
Veenu Kamra and Achim Dreher, Senior Member, IEEE
}

\begin{abstract}
In this paper, the discrete mode matching (DMM) method is extended to characterize multilayered transmission line structures with inhomogeneous anisotropic dielectric layers using only 1D discretization. We have thoroughly discussed the procedure to analyze hybrid dielectric waveguides with dielectric strips and/or microstrips, i.e., metallization at the interfaces. The analysis of the structures is based on the full-wave equivalent circuit approach, which gives the generalized relation of the field components of the multilayered structure and the corresponding system equation. Accurate results are obtained with very less computational effort. The dispersion curves and the electric field intensity distributions are presented for various dielectric waveguides and validated with the commercial software.
\end{abstract}

Index Terms-Channel waveguides, microstrips, anisotropic, inhomogeneous media, optical waveguides.

\section{INTRODUCTION}

$\mathbf{P}$ LANAR dielectric waveguides are widely used in microwave and millimeter-wave circuits using non-radiative dielectric (NRD) waveguide integrated technology [1], substrate integrated technology [2], and also in modern integrated optics [3]. This paper focuses on multilayered structures consisting of homogeneous or inhomogeneous dielectric layers with or without metallization in the interfaces. Various hybrid dielectric waveguides, which are multilayered waveguides with dielectric strips and microstrips, are shown in Fig. 1. These inhomogeneities are fabricated in the fibers or optical waveguides to provide the desired confinement. Here the problem is to characterize the media where the dielectric constant is dependent on space. It is desired by the design engineers that the dielectric waveguides must be accurately modeled with efficient numerical procedures so that they have precise value of propagation constants for these complex multilayered structures.

Several approaches are already used to precisely compute the propagation constants of the inhomogeneous waveguides, like Fourier decomposition in [4], method of lines (MoL) in [5], [6], finite element method in [7], variational method in [8] and plane wave expansion method in [9]. 24 years ago, a method has been developed for the simple full-wave

The authors are with the German Aerospace Center (DLR), Institute of Communications and Navigation, Oberpfaffenhofen, D-82234 Wessling, Germany (e-mail: veenu.kamra@dlr.de; achim.dreher@dlr.de).

Manuscript received ..

Digital Object Identifier ..

This paper is an expanded version of a conference paper presented at IEEE MTT-S IMS 2019, Boston, MA. analysis of waveguides, microstrips and patch antennas with stratified media, known as the discretized mode matching (DMM) method [10]. It was originally developed to overcome the disadvantage of the MoL, i.e., the approximation of the differential operators and calculating the eigenvalues of the corresponding matrices which introduces additional errors in the computation. The DMM method uses the exact eigenvalues of the waveguide modes which are dependent on the lateral boundary conditions. Thus, it reduces the computational effort due to unnecessary approximations. The Green's function (or system equation) is derived using a full-wave equivalent circuit (FWEC) in the spectral domain [11]. The application of the DMM for the analysis of transmission lines on planar, quasi-planar, cylindrical and quasi-cylindrical isotropic and/or anisotropic substrate with homogeneous dielectric layers has been described earlier in [12]-[16]. The literature [16] also illustrates the variation of the normalized propagation constant with respect to the number of discretization lines in cylindrical coordinate system.

Our recent work [17] extends the DMM method and explains the derivation of the hybrid-matrix elements for the inhomogeneous dielectric layers. We have done analysis for several inhomogeneous waveguides in that paper and also seen the effect of anisotropy in channel waveguides. In this paper, we examine more in-depth and clear analysis with inhomogeneous structures. We also consider metallization between the dielectric layers and take into account the interfaces which are not in accordance with the coordinate axes. Then we use the formulation to generate the Green's function or the system equation of the multilayered structure under analysis.

In section II, we introduce the detailed theory for the analysis of inhomogeneous dielectric layers. The full-wave analysis is obtained using summation of both kind of modes, transversal electric and transversal magnetic modes. We also discuss the various cases where the derived hybrid matrices can be useful. In section III, we discuss various numerical results and validate them with open literature and/or commercial software ANSYS HFSS.

\section{THEORY}

\section{A. Field Equations}

We consider an arbitrary planar multilayered structure with homogeneous and inhomogeneous dielectric layers. The direction of the wave propagation is taken in $z$-axis and the 


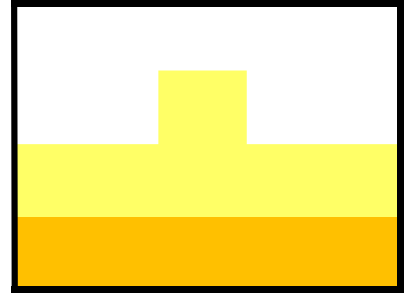

(a)

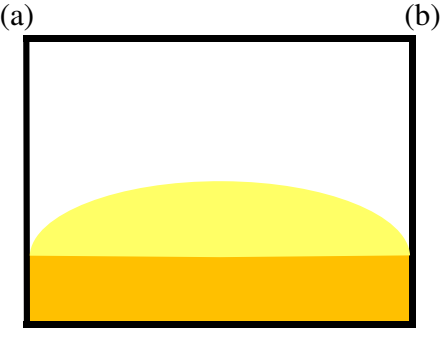

(c)

Fig. 1: Hybrid dielectric waveguide structures. Black line: metallization; green, orange, yellow, white: different dielectric regions. (a) Waveguide with dielectric strip. (b) Waveguide with dielectric strip and microstrip. (c) Waveguide with quasi-planar interface.

cross-section in the $x y$-plane as shown in Fig. 2. The figure consists of metallic strip at $k^{\text {th }}$ interface. The inhomogeneous layer $k$ contains an abrupt transition in the material properties. Therefore, the space dependent permittivity $\left(\overline{\bar{\varepsilon}}=\varepsilon_{0} \overline{\bar{\varepsilon}}_{r}\right)$ and permeability $\left(\overline{\bar{\mu}}=\mu_{0} \overline{\bar{\mu}}_{r}\right)$ tensors of the dielectric layer can be written with

$$
\begin{gathered}
\overline{\bar{\varepsilon}}_{r}=\left(\begin{array}{ccc}
\varepsilon_{x}(x, y) & 0 & 0 \\
0 & \varepsilon_{y}(x, y) & 0 \\
0 & 0 & \varepsilon_{z}(x, y)
\end{array}\right), \\
\overline{\bar{\mu}}_{r}=\left(\begin{array}{ccc}
\mu_{x}(x, y) & 0 & 0 \\
0 & \mu_{y}(x, y) & 0 \\
0 & 0 & \mu_{z}(x, y)
\end{array}\right) .
\end{gathered}
$$

The source-free second order curl-curl equations are obtained for electric and magnetic fields as

$$
\begin{aligned}
& \nabla \times \overline{\bar{\mu}}_{r}^{-1} \cdot \nabla \times \vec{E}(x, y, z)-\overline{\bar{\varepsilon}}_{r} \cdot \vec{E}(x, y, z)=0, \\
& \nabla \times \overline{\bar{\varepsilon}}_{r}^{-1} \cdot \nabla \times \vec{H}(x, y, z)-\overline{\bar{\mu}}_{r} \cdot \vec{H}(x, y, z)=0,
\end{aligned}
$$

where $\vec{E}$ and $\vec{H}$ represent electric and magnetic field vectors. In rectangular coordinate system, we write

$$
\vec{E}(x, y, z)=E_{x}(x, y, z) \hat{x}+E_{y}(x, y, z) \hat{y}+E_{z}(x, y, z) \hat{z},
$$

with $\hat{x}, \hat{y}$ and $\hat{z}$ denoting the unit vectors along $x, y$ and $z$ coordinates, respectively. Here, all the space variables are normalized by the free space wave number $k_{0}$, the time factor is suppressed and $\eta_{0} \vec{H}$ is replaced by $\vec{H}$, where $\eta_{0}=\sqrt{\mu_{0} / \varepsilon_{0}}$ is the intrinsic impedance of the free space. We can take a large number of layers in $y$-direction, which removes the $y$-dependance of the material properties.

On expanding (3)-(4) into 6 scalar differential equations, we see that each obtained equation is at least coupled to another one. It is a well-known fact that hybrid modes are a combination of TE and TM modes. Therefore, we consider $\mathrm{TE}^{x}\left(E_{x}^{\mathrm{TE}}=0\right)$ and $\mathrm{TM}^{x}\left(H_{x}^{\mathrm{TM}}=0\right)$ modes to obtain the uncoupled scalar differential equations and take $\psi=E_{y}^{\mathrm{TE}}, H_{y}^{\mathrm{TM}}$ to be two independent field components. We obtain the differential equation for $\mathrm{TE}^{x}$ modes as

$\frac{\partial^{2}}{\partial y^{2}} E_{y}^{\mathrm{TE}}+\mu_{x} \frac{\varepsilon_{z}}{\varepsilon_{y}} \frac{\partial}{\partial x} \frac{1}{\mu_{z}} \frac{\partial}{\partial x} E_{y}^{\mathrm{TE}}+\mu_{x} \varepsilon_{z} E_{y}^{\mathrm{TE}}-\frac{\varepsilon_{z}}{\varepsilon_{y}} k_{z}^{2} E_{y}^{\mathrm{TE}}=0$.

Similarly, we obtain the differential equation for $\mathrm{TM}^{x}$ modes as

$\frac{\partial^{2}}{\partial y^{2}} H_{y}^{\mathrm{TM}}+\varepsilon_{x} \frac{\mu_{z}}{\mu_{y}} \frac{\partial}{\partial x} \frac{1}{\varepsilon_{z}} \frac{\partial}{\partial x} H_{y}^{\mathrm{TM}}+\mu_{z} \varepsilon_{x} H_{y}^{\mathrm{TM}}-\frac{\mu_{z}}{\mu_{y}} k_{z}^{2} H_{y}^{\mathrm{TM}}=0$.

We can calculate all the electric and magnetic field components with the following set of equations:

$$
\begin{gathered}
E_{x}=\frac{1}{k_{z}}\left(\frac{\partial}{\partial x} \frac{1}{\varepsilon_{z}} \frac{\partial}{\partial x} H_{y}^{\mathrm{TM}}+\mu_{y} H_{y}^{\mathrm{TM}}\right) \\
H_{x}=-\frac{1}{k_{z}}\left(\frac{\partial}{\partial x} \frac{1}{\mu_{z}} \frac{\partial}{\partial x} E_{y}^{\mathrm{TE}}+\varepsilon_{y} E_{y}^{\mathrm{TE}}\right) \\
E_{z}=-\frac{j}{k_{z}} \frac{\varepsilon_{y}}{\varepsilon_{z}} \frac{\partial}{\partial y} E_{y}^{\mathrm{TE}}-\frac{j}{\varepsilon_{z}} \frac{\partial}{\partial x} H_{y}^{\mathrm{TM}} \\
H_{z}=\frac{j}{\mu_{z}} \frac{\partial}{\partial x} E_{y}^{\mathrm{TE}}-\frac{j}{k_{z}} \frac{\mu_{y}}{\mu_{z}} \frac{\partial}{\partial y} H_{y}^{\mathrm{TM}} \\
E_{y}=E_{y}^{\mathrm{TE}}+\frac{1}{\varepsilon_{z} k_{z}} \frac{\partial}{\partial y} \frac{\partial}{\partial x} H_{y}^{\mathrm{TM}} \\
H_{y}=H_{y}^{\mathrm{TM}}-\frac{1}{\mu_{z} k_{z}} \frac{\partial}{\partial y} \frac{\partial}{\partial x} E_{y}^{\mathrm{TE}} .
\end{gathered}
$$

\section{B. Discrete Mode Matching Method}

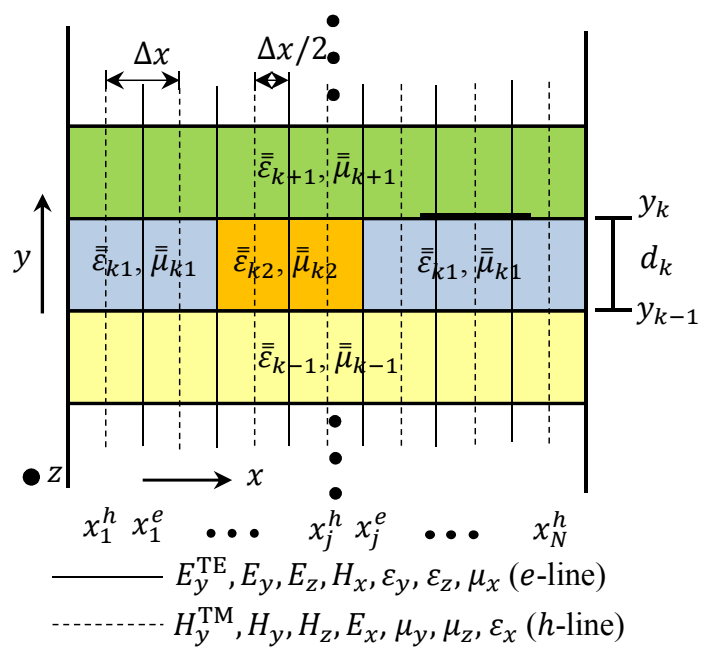

Fig. 2: Discretization scheme for the waveguide structure with inhomogeneous dielectric layers.

We consider that the transmission lines are infinite in the propagation direction. Therefore, the method employs only 1D discretization along the horizontal tangential direction ( $x$-axis) for the analysis and takes the analytical solution in the perpendicular direction ( $y$-axis). The structure is discretized 
using $e$ - and $h$-lines, which give the position of $E_{y}^{\mathrm{TE}}$ and $H_{y}^{\mathrm{TM}}$, respectively. Fig. 2 shows the equidistant discretization scheme used for the inhomogeneous dielectric layers bounded with electric walls. We take the distance between the edge of the metallization and $e$-line to be $0.25 \Delta x$. We can relate the spatial domain field components in the spectral domain using the relation in matrix form

$$
\psi=T S \widetilde{\psi}
$$

or we write

$$
\begin{gathered}
\boldsymbol{E}_{y}^{\mathrm{TE}}=\boldsymbol{T}_{e} \boldsymbol{S}_{e} \widetilde{\boldsymbol{E}}_{y}^{\mathrm{TE}} \\
\boldsymbol{H}_{y}^{\mathrm{TM}}=\boldsymbol{T}_{h} \boldsymbol{S}_{h} \widetilde{\boldsymbol{H}}_{y}^{\mathrm{TM}} .
\end{gathered}
$$

Here $\widetilde{\psi}$ represents the column matrix of field component in spectral domain containing all the modal values, $\psi$ represents the column matrix of field component in space domain, $\boldsymbol{T}_{e, h}$ gives the transformation matrices and $\boldsymbol{S}_{e, h}$ denotes additional matrices which account for inhomogeneity in the dielectric layer (subscript $e$ is for $e$-lines and subscript $h$ is for $h$-lines). We calculate the transformation matrices depending on the conditions used to bound the lateral domain which consists of eigensolutions with exact eigenvalues.

We can easily identify the locations of the other field components with the help of the field relations (8)-(11). Fig. 2 describes the positions of all the field components and material properties. Therefore, the relation of the other field components between the space domain and the spectral domain in matrix form can be summarized as

$$
\begin{aligned}
\boldsymbol{E}_{x} & =\boldsymbol{T}_{h} \widetilde{\boldsymbol{E}}_{x} \\
\boldsymbol{E}_{y} & =\boldsymbol{T}_{e} \widetilde{\boldsymbol{E}}_{y} \\
\boldsymbol{E}_{z} & =\boldsymbol{T}_{e} \widetilde{\boldsymbol{E}}_{y} \\
\boldsymbol{H}_{x} & =\boldsymbol{T}_{e} \widetilde{\boldsymbol{H}}_{y} \\
\boldsymbol{H}_{y} & =\boldsymbol{T}_{h} \widetilde{\boldsymbol{H}}_{y} \\
\boldsymbol{H}_{z} & =\boldsymbol{T}_{h} \widetilde{\boldsymbol{H}}_{z} .
\end{aligned}
$$

On using the continuity equation of magnetic fields on the interface, we get the relation for the current densities as

$$
\begin{aligned}
\boldsymbol{J}_{x} & =\boldsymbol{T}_{h} \widetilde{\boldsymbol{J}}_{x} \\
\boldsymbol{J}_{z} & =\boldsymbol{T}_{e} \widetilde{\boldsymbol{J}}_{z} .
\end{aligned}
$$

The discontinuities in layer $k$ are aligned with the $e$-lines, therefore the material properties on these lines change accordingly:

$$
\begin{aligned}
\varepsilon_{y} & =\frac{\varepsilon_{y 1}+\varepsilon_{y 2}}{2} \\
\varepsilon_{z} & =\frac{\varepsilon_{z 1}+\varepsilon_{z 2}}{2} \\
\mu_{x} & =\frac{\mu_{x 1}+\mu_{x 2}}{2} .
\end{aligned}
$$

The reason for taking the arithmetic mean of the material properties at the discontinuities is well explained in [6]. Due to inhomogeneity in the dielectric layer, we get the diagonal matrix of varying material properties along the discretization in $x$-direction. These matrices can be converted into spectral domain using the relation

$$
\begin{aligned}
\bar{\varepsilon}_{e, h} & =\boldsymbol{T}_{e, h}^{-1} \varepsilon_{e, h} \boldsymbol{T}_{e, h} \\
\overline{\boldsymbol{\mu}}_{e, h} & =\boldsymbol{T}_{e, h}^{-1} \boldsymbol{\mu}_{e, h} \boldsymbol{T}_{e, h} .
\end{aligned}
$$

On transforming (6) and (7) using (14), we get the relations

$$
\begin{gathered}
\boldsymbol{Q}_{e}=\overline{\boldsymbol{\mu}}_{x e} \overline{\boldsymbol{\varepsilon}}_{z e} \overline{\boldsymbol{\varepsilon}}_{y e}^{-1} \boldsymbol{k}_{x} \overline{\boldsymbol{\mu}}_{z h}^{-1} \boldsymbol{k}_{x}-\overline{\boldsymbol{\mu}}_{x e} \overline{\boldsymbol{\varepsilon}}_{z e}+\overline{\boldsymbol{\varepsilon}}_{z e} \overline{\boldsymbol{\varepsilon}}_{y e}^{-1} k_{z}^{2}, \\
\boldsymbol{Q}_{h}=\overline{\boldsymbol{\varepsilon}}_{x h} \overline{\boldsymbol{\mu}}_{z h} \overline{\boldsymbol{\mu}}_{y h}^{-1} \boldsymbol{k}_{x} \overline{\boldsymbol{\varepsilon}}_{z e}^{-1} \boldsymbol{k}_{x}-\overline{\boldsymbol{\mu}}_{z h} \overline{\boldsymbol{\varepsilon}}_{x h}+\overline{\boldsymbol{\mu}}_{z h} \overline{\boldsymbol{\mu}}_{y h}^{-1} k_{z}^{2},
\end{gathered}
$$

where $\boldsymbol{k}_{x}$ denotes the eigenvalues.

Now, there is a need to solve the eigenvalue problems

$$
\boldsymbol{Q}_{e} \boldsymbol{S}_{e}=\boldsymbol{S}_{e} \boldsymbol{k}_{y e}^{2} \text { and } \boldsymbol{Q}_{h} \boldsymbol{S}_{h}=\boldsymbol{S}_{h} \boldsymbol{k}_{y h}^{2},
$$

to obtain the ordinary differential equation

$$
\left(\frac{d^{2}}{d y^{2}}-\boldsymbol{k}_{y e, h}^{2}\right) \widetilde{\boldsymbol{\psi}}_{e, h}=0 .
$$

On solving the separated equation (33), we get the solution within the arbitrary layer $k$ as

$$
\begin{aligned}
\widetilde{\boldsymbol{E}}_{y_{k}}^{\mathrm{TE}} & =\boldsymbol{A}_{k} e^{-\boldsymbol{k}_{y e_{k}} y}+\boldsymbol{B} e^{\boldsymbol{k}_{y_{k}} y}, \\
\widetilde{\boldsymbol{H}}_{y_{k}}^{\mathrm{TM}} & =\boldsymbol{C}_{k} e^{-\boldsymbol{k}_{y h_{k}} y}+\boldsymbol{D}_{k} e^{\boldsymbol{k}_{y h_{k}} y} .
\end{aligned}
$$

After discretizing the structure and transforming the field components (8)-(11) according to (15)-(22), we get the tangential field components in spectral domain as

$$
\begin{gathered}
\widetilde{\boldsymbol{E}}_{x}=\frac{1}{k_{z}}\left(\left(-j \boldsymbol{k}_{x}\right) \overline{\boldsymbol{\varepsilon}}_{z e}^{-1}\left(-j \boldsymbol{k}_{x}\right)+\overline{\boldsymbol{\mu}}_{y h}\right) \boldsymbol{S}_{h} \widetilde{\boldsymbol{H}}_{y}^{\mathrm{TM}} \\
\widetilde{\boldsymbol{H}}_{x}=-\frac{1}{k_{z}}\left(\left(-j \boldsymbol{k}_{x}\right) \overline{\boldsymbol{\mu}}_{z h}^{-1}\left(-j \boldsymbol{k}_{x}\right)+\overline{\boldsymbol{\varepsilon}}_{y e}\right) \boldsymbol{S}_{e} \widetilde{\boldsymbol{E}}_{y}^{\mathrm{TE}} \\
\widetilde{\boldsymbol{E}}_{z}=-\frac{j}{k_{z}} \overline{\boldsymbol{\varepsilon}}_{y e} \overline{\boldsymbol{\varepsilon}}_{z e}^{-1} \frac{\partial}{\partial y} \boldsymbol{S}_{e} \widetilde{\boldsymbol{E}}_{y}^{\mathrm{TE}}-j \overline{\boldsymbol{\varepsilon}}_{z e}^{-1}\left(-j \boldsymbol{k}_{x}\right) \boldsymbol{S}_{h} \widetilde{\boldsymbol{H}}_{y}^{\mathrm{TM}} \\
\widetilde{\boldsymbol{H}}_{z}=j \overline{\boldsymbol{\mu}}_{z h}^{-1}\left(-j \boldsymbol{k}_{x}\right) \boldsymbol{S}_{e} \widetilde{\boldsymbol{E}}_{y}^{\mathrm{TE}}-\frac{j}{k_{z}} \overline{\boldsymbol{\mu}}_{y h} \overline{\boldsymbol{\mu}}_{z h}^{-1} \frac{\partial}{\partial y} \boldsymbol{S}_{h} \widetilde{\boldsymbol{H}}_{y}^{\mathrm{TM}} .
\end{gathered}
$$

On using (34)-(39), we rewrite the tangential field components for the layer $k$ in matrix form as

$$
\left[\begin{array}{c}
\widetilde{\boldsymbol{E}}_{x_{k}} \\
\widetilde{\boldsymbol{H}}_{x_{k}} \\
\widetilde{\boldsymbol{E}}_{z_{k}} \\
\widetilde{\boldsymbol{H}}_{z_{k}}
\end{array}\right]=\left[\begin{array}{cccc}
\widetilde{\boldsymbol{Q}}_{x_{k}}^{A} & \widetilde{\boldsymbol{Q}}_{x_{k}}^{B} & \widetilde{\boldsymbol{Q}}_{x_{k}}^{C} & \widetilde{\boldsymbol{Q}}_{x_{k}}^{D} \\
\widetilde{\boldsymbol{G}}_{x_{k}}^{A} & \widetilde{\boldsymbol{G}}_{x_{k}}^{B} & \widetilde{\boldsymbol{G}}_{x_{k}}^{C} & \widetilde{\boldsymbol{G}}_{x_{k}}^{D} \\
\widetilde{\boldsymbol{Q}}_{z_{k}}^{A} & \widetilde{\boldsymbol{Q}}_{z_{k}}^{B} & \widetilde{\boldsymbol{Q}}_{z_{k}}^{C} & \widetilde{\boldsymbol{Q}}_{z_{k}}^{D} \\
\widetilde{\boldsymbol{G}}_{z_{k}}^{A} & \widetilde{\boldsymbol{G}}_{z_{k}}^{B} & \widetilde{\boldsymbol{G}}_{z_{k}}^{C} & \widetilde{\boldsymbol{G}}_{z_{k}}^{D}
\end{array}\right]\left[\begin{array}{c}
\boldsymbol{A}_{k} \\
\boldsymbol{B}_{k} \\
\boldsymbol{C}_{k} \\
\boldsymbol{D}_{k}
\end{array}\right] .
$$

Now to obtain the relation between the fields at the interfaces $k-1$ and $k$ bounding the layer $k$, we write

$$
\begin{aligned}
& {\left[\begin{array}{c}
\widetilde{\boldsymbol{E}}_{k-1} \\
\widetilde{\boldsymbol{H}}_{k-1}
\end{array}\right]=\widetilde{\boldsymbol{M}}_{k-1} \boldsymbol{F}, \quad\left[\begin{array}{c}
\widetilde{\boldsymbol{E}}_{k} \\
\widetilde{\boldsymbol{H}}_{k}
\end{array}\right]=\widetilde{\boldsymbol{M}}_{k} \boldsymbol{F}, } \\
& \widetilde{\boldsymbol{M}}_{k}=\left[\begin{array}{cccc}
\widetilde{\boldsymbol{Q}}_{x_{k}}^{A} & \widetilde{\boldsymbol{Q}}_{x_{k}}^{B} & \widetilde{\boldsymbol{Q}}_{x_{k}}^{C} & \widetilde{\boldsymbol{Q}}_{x_{k}}^{D} \\
\widetilde{\boldsymbol{Q}}_{z_{k}}^{A} & \widetilde{\boldsymbol{Q}}_{z_{k}}^{B} & \widetilde{\boldsymbol{Q}}_{z_{k}}^{C} & \widetilde{\boldsymbol{Q}}_{z_{k}}^{D} \\
\widetilde{\boldsymbol{G}}_{z_{k}}^{A} & \widetilde{\boldsymbol{G}}_{z_{k}}^{B} & \widetilde{\boldsymbol{G}}_{z_{k}}^{C} & \widetilde{\boldsymbol{G}}_{z_{k}}^{D} \\
-\widetilde{\boldsymbol{G}}_{x_{k}}^{A} & -\widetilde{\boldsymbol{G}}_{x_{k}}^{B} & -\widetilde{\boldsymbol{G}}_{x_{k}}^{C} & -\widetilde{\boldsymbol{G}}_{x_{k}}^{D}
\end{array}\right]\left[\begin{array}{c}
\boldsymbol{A}_{k} \\
\boldsymbol{B}_{k} \\
\boldsymbol{C}_{k} \\
\boldsymbol{D}_{k}
\end{array}\right],
\end{aligned}
$$




$$
\left[\begin{array}{c}
\widetilde{\boldsymbol{J}}_{1} \\
\widetilde{\boldsymbol{J}}_{2} \\
\widetilde{\boldsymbol{J}}_{3} \\
\vdots \\
\widetilde{\boldsymbol{J}}_{M-1} \\
\widetilde{\boldsymbol{J}}_{M}
\end{array}\right]=\left[\begin{array}{cccccc}
\widetilde{\boldsymbol{L}}_{11} & \widetilde{\boldsymbol{L}}_{12} & \mathbf{0} & \mathbf{0} & \cdots & \mathbf{0} \\
\widetilde{\boldsymbol{L}}_{21} & \widetilde{\boldsymbol{L}}_{22} & \widetilde{\boldsymbol{L}}_{23} & \mathbf{0} & \cdots & \mathbf{0} \\
\mathbf{0} & \widetilde{\boldsymbol{L}}_{32} & \widetilde{\boldsymbol{L}}_{33} & \widetilde{\boldsymbol{L}}_{34} & \cdots & \mathbf{0} \\
\vdots & \cdots & \ddots & \ddots & \cdots & \vdots \\
\mathbf{0} & \cdots & \mathbf{0} & \widetilde{\boldsymbol{L}}_{M-1, M-2} & \widetilde{\boldsymbol{L}}_{M-1, M-1} & \widetilde{\boldsymbol{L}}_{M-1, M} \\
\mathbf{0} & \cdots & \mathbf{0} & \mathbf{0} & \widetilde{\boldsymbol{L}}_{M, M-1} & \widetilde{\boldsymbol{L}}_{M, M}
\end{array}\right]\left[\begin{array}{c}
\widetilde{\boldsymbol{E}}_{1} \\
\widetilde{\boldsymbol{E}}_{2} \\
\widetilde{\boldsymbol{E}}_{3} \\
\vdots \\
\widetilde{\boldsymbol{E}}_{M-1} \\
\widetilde{\boldsymbol{E}}_{M}
\end{array}\right]
$$

and take the notations of the fields and coefficients in matrix form as

$$
\widetilde{\boldsymbol{E}}_{k}=\left[\begin{array}{c}
\widetilde{\boldsymbol{E}}_{x_{k}} \\
\widetilde{\boldsymbol{E}}_{z_{k}}
\end{array}\right], \widetilde{\boldsymbol{H}}_{k}=\left[\begin{array}{c}
\widetilde{\boldsymbol{H}}_{z_{k}} \\
-\widetilde{\boldsymbol{H}}_{x_{k}}
\end{array}\right], \boldsymbol{F}=\left[\begin{array}{c}
\boldsymbol{A}_{k} \\
\boldsymbol{B}_{k} \\
\boldsymbol{C}_{k} \\
\boldsymbol{D}_{k}
\end{array}\right] .
$$

After removing the unknown column matrix $\boldsymbol{F}$ from (41), we get the relation for the field components at the interfaces of the layer $k$ with normalized thickness $d_{k}$ as

$$
\left[\begin{array}{c}
\widetilde{\boldsymbol{E}}_{k-1} \\
\widetilde{\boldsymbol{H}}_{k-1}
\end{array}\right]=\widetilde{\boldsymbol{K}}_{k}\left[\begin{array}{c}
\widetilde{\boldsymbol{E}}_{k} \\
\widetilde{\boldsymbol{H}}_{k}
\end{array}\right],
$$

with the hybrid matrix

$$
\widetilde{\boldsymbol{K}}_{k}=\widetilde{\boldsymbol{M}}_{k-1} \widetilde{\boldsymbol{M}}_{k}^{-1}=\left[\begin{array}{ll}
\widetilde{\boldsymbol{V}}_{k} & \widetilde{\boldsymbol{Z}}_{k} \\
\widetilde{\boldsymbol{Y}}_{k} & \widetilde{\boldsymbol{B}}_{k}
\end{array}\right] .
$$

We can take into account the multiple dielectric layers by simple matrix multiplication. After getting hybrid matrices for each layer, we must apply continuity equations to match the fields at the interfaces. Then the Green's function $(\widetilde{\boldsymbol{G}})$ can be found in spectral domain after applying the theory of full-wave equivalent circuit and network analysis technique according to [11]. The system equation takes the form

$$
\widetilde{\boldsymbol{G}} \widetilde{\boldsymbol{J}}=\widetilde{\boldsymbol{E}},
$$

where $\widetilde{\boldsymbol{J}}$ and $\widetilde{\boldsymbol{E}}$ represent electric current density and electric field in the interfaces, respectively. There are no currents on the interfaces of the waveguides, therefore the system equation becomes

$$
\widetilde{\boldsymbol{L}} \widetilde{\boldsymbol{E}}=0,
$$

by applying the boundary conditions at the interfaces. Consequently, the propagation constant can be found on solving the indirect eigenvalue problem (47) with

$$
\operatorname{det}(\widetilde{\boldsymbol{L}})=0 \text { where } \widetilde{\boldsymbol{L}}=\widetilde{\boldsymbol{G}}^{-1} .
$$

1) Interface with Metallization: Now consider the case when metallization is present on the interfaces of the structure. We take the metallization to be infinitely thin. The system matrix with several metallized interfaces $(M)$ can be represented by (49) or by

$$
\widetilde{\boldsymbol{J}}=\widetilde{\boldsymbol{L}} \widetilde{\boldsymbol{E}}
$$

The elements of $\widetilde{\boldsymbol{L}}$ matrix can be found using the relations:

$$
\begin{aligned}
\widetilde{\boldsymbol{L}}_{k, k-1} & =-\widetilde{\boldsymbol{Z}}_{k}^{-1} \\
\widetilde{\boldsymbol{L}}_{k, k} & =\widetilde{\boldsymbol{B}}_{k+1} \widetilde{\boldsymbol{Z}}_{k+1}^{-1}+\widetilde{\boldsymbol{Z}}_{k}^{-1} \widetilde{\boldsymbol{V}}_{k} \\
\widetilde{\boldsymbol{L}}_{k, k+1} & =\widetilde{\boldsymbol{Y}}_{k+1}-\widetilde{\boldsymbol{B}}_{k+1} \widetilde{\boldsymbol{Z}}_{k+1}^{-1} \widetilde{\boldsymbol{V}}_{k+1} .
\end{aligned}
$$

Now, we transform the field components and current densities in (46) into the space domain using (17)-(24) and get

$$
\left[\begin{array}{ll}
\boldsymbol{G}_{x x} & \boldsymbol{G}_{x z} \\
\boldsymbol{G}_{z x} & \boldsymbol{G}_{z z}
\end{array}\right]\left[\begin{array}{l}
\boldsymbol{J}_{x} \\
\boldsymbol{J}_{z}
\end{array}\right]=\left[\begin{array}{l}
\boldsymbol{E}_{x} \\
\boldsymbol{E}_{z}
\end{array}\right] \quad \text { or } \quad \boldsymbol{G} \boldsymbol{J}=\boldsymbol{E}
$$

where

$$
\left[\begin{array}{ll}
\boldsymbol{G}_{x x} & \boldsymbol{G}_{x z} \\
\boldsymbol{G}_{z x} & \boldsymbol{G}_{z z}
\end{array}\right]=\left[\begin{array}{cc}
\boldsymbol{T}_{h} & 0 \\
0 & \boldsymbol{T}_{e}
\end{array}\right]\left[\begin{array}{cc}
\widetilde{\boldsymbol{G}}_{x x} & \widetilde{\boldsymbol{G}}_{x z} \\
\widetilde{\boldsymbol{G}}_{z x} & \widetilde{\boldsymbol{G}}_{z z}
\end{array}\right]\left[\begin{array}{cc}
\boldsymbol{T}_{h}^{-1} & 0 \\
0 & \boldsymbol{T}_{e}^{-1}
\end{array}\right] .
$$

Here $\boldsymbol{G}=\boldsymbol{L}^{-1}$ denotes the Green's function in space domain.

After obtaining the system equation, the boundary conditions must be applied. The conditions state that the tangential electric field components must vanish on the metallizations and the electric currents outside that region. We obtain the reduced system equation as

$$
\boldsymbol{G}_{\text {red }} \boldsymbol{J}_{\text {red }}=0 \quad \text { or } \quad \boldsymbol{L}_{\text {red }} \boldsymbol{E}_{\text {red }}=0,
$$

where $\boldsymbol{J}_{\text {red }}$ contains the tangential current density components located on the metallizations, while $\boldsymbol{E}_{\text {red }}$ contains the tangential electric field components located outside the metallization. We can solve it as an indirect eigenvalue problem with

$$
\operatorname{det}\left(\boldsymbol{G}_{\text {red }}\right)=0 \quad \text { or } \quad \operatorname{det}\left(\boldsymbol{L}_{\text {red }}\right)=0
$$

to find the propagation constants for the transmission lines with metallization in the interfaces. $\boldsymbol{L}_{\text {red }}$ is equivalent to $\boldsymbol{L}$ in waveguides, as there are no currents on the interfaces.

2) Quasi-planar Interface: When the interfaces between the layers are not constant according to the coordinate system then there are two ways to deal with such type of problems. One way is to divide the non-planar dielectric layer into several layers with changing dielectric strip width as shown in Fig. 3 a. This will lead us to deal with inhomogeneous layers which has been explained in earlier sections. The other way is to calculate tangential field components at the actual interface as shown in Fig. 3b.

In the second way we deal with non-planar interfaces within the dielectric directly, therefore the whole analysis should be done in space domain [14]. We assume that the quasi planar nature of the layers at the interfaces is in $x$-direction and the cross-section is constant in $z$-direction. We need to calculate 


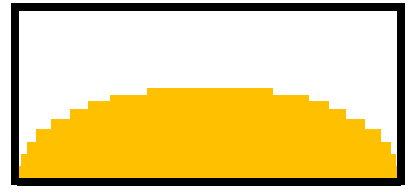

(a)

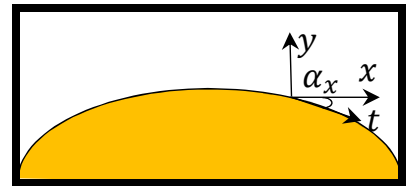

(b)
Fig. 3: Waveguide with quasi-planar interface. (a) Changing strip width. (b) Smooth interface.



Fig. 4: Convergence behavior of normalized propagation constant for insulated image waveguide.

the tangential field components at the interfaces. In this case, the components no longer remain in the $x$-direction as in the previous case, but $E_{z}$ and $H_{z}$ are tangential to the interfaces along the whole $x$-direction. Therefore the other tangential fields become $E_{t x}$ and $H_{t x}$, and are represented as

$$
\begin{aligned}
& E_{t x_{k}}=E_{x_{k}} \cos \alpha_{x^{h}}+\bar{E}_{y_{k}} \sin \alpha_{x^{h}}, \\
& H_{t x_{k}}=H_{x_{k}} \cos \alpha_{x^{e}}+\bar{H}_{y_{k}} \sin \alpha_{x^{e}} .
\end{aligned}
$$

Here $\alpha_{x(e, h)}$ represents the inclination angle at the interface with the $x$-axis.

From Fig. 2, we can say that for 1D discretization $E_{y}^{\mathrm{TE}}$, $E_{z}, E_{y}$ and $H_{x}$ (or $H_{y}^{\mathrm{TM}}, H_{z}, H_{y}$ and $E_{x}$ ) components are sampled at the same position. To determine $E_{t x}$, we must calculate $E_{x}$ and $E_{y}$ at the same point. Therefore, $\bar{E}_{y}$ is used in (56) which is the mean of the adjacent sampled values of $E_{y}$. Similarly, $\bar{H}_{y}$ is calculated from the mean of the adjacent values of $H_{y}$. Then, (43) changes according to

$$
\boldsymbol{E}_{k}=\left[\begin{array}{c}
\boldsymbol{E}_{t x_{k}} \\
\boldsymbol{E}_{z_{k}}
\end{array}\right], \boldsymbol{H}_{k}=\left[\begin{array}{c}
\boldsymbol{H}_{z_{k}} \\
-\boldsymbol{H}_{t x_{k}}
\end{array}\right] \text {. }
$$

\section{NUMERICAL RESULTS}

\section{A. Convergence for Insulated Image Waveguide}

First, we have taken the insulated image waveguide, shown in the inset of Fig 4, for the analysis. It is widely used for optical communication. We have analyzed the waveguide with

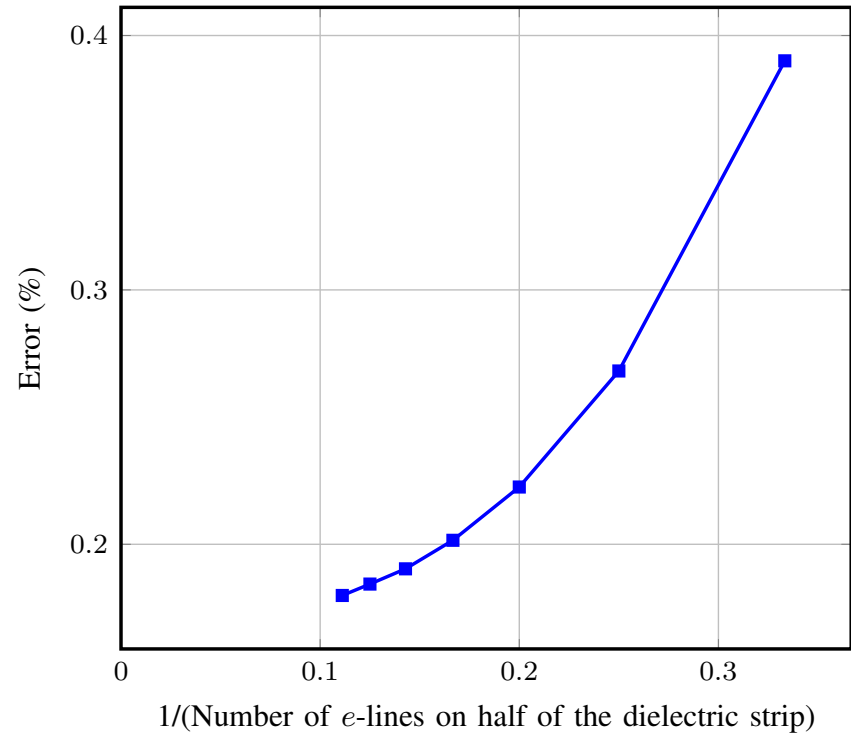

Fig. 5: Relative error of DMM results with respect to HFSS solution.

$\varepsilon_{r 1}=1.5, \varepsilon_{r 2}=3.8, \varepsilon_{r 3}=1, a / d_{2}=13.5, b / d_{2}=8$, $d_{1} / d_{2}=0.5, w / d=2.25$ and $d_{2}=1.5$. The two bottom layers are combined together and the resultant hybrid matrix is obtained as

$$
\widetilde{\boldsymbol{K}}_{e q}=\widetilde{\boldsymbol{K}}_{1} \widetilde{\boldsymbol{K}}_{2}=\left[\begin{array}{ll}
\widetilde{\boldsymbol{V}}_{e q} & \widetilde{\boldsymbol{Z}}_{e q} \\
\widetilde{\boldsymbol{Y}}_{e q} & \widetilde{\boldsymbol{B}}_{e q}
\end{array}\right] .
$$

From network analysis techniques, we simply obtain the system equation as

$$
\widetilde{\boldsymbol{L}} \widetilde{\boldsymbol{E}}=0 \text {, where } \widetilde{\boldsymbol{L}}=\widetilde{\boldsymbol{Z}}_{e q}^{-1} \widetilde{\boldsymbol{V}}_{e q}+\widetilde{\boldsymbol{B}}_{3} \widetilde{\boldsymbol{Z}}_{3}^{-1} .
$$

The first term of the admittance $\left(\widetilde{\boldsymbol{Z}}_{e q}^{-1} \widetilde{\boldsymbol{V}}_{e q}\right)$ denotes the admittance of the grounded bottom layer and the second term $\left(\widetilde{\boldsymbol{B}}_{3} \widetilde{\boldsymbol{Z}}_{3}^{-1}\right)$ denotes the admittance of the closed top layer.

Fig. 4 shows the convergence of the propagation constant (normalized by $k_{0}$ ) of the fundamental mode with increasing number of $e$-lines used for discretization of half of the dielectric strip with $\varepsilon_{r 2}$. The increase in the discretization lines is equivalent to the increasing number of modes used for the field expansion due to the Nyquist-Shannon sampling theorem. We have computed the values for upto 9 $e$-lines because results do not vary much with increment in discretization lines after certain limit. We can see from the figure that the results with 7,8 and $9 e$-lines are very close to each other and within a small number of discretization lines we can reach a high level of accuracy. An extrapolation to the exact value given by an infinite number of lines and modes $(1 /$ (Number of $e$-lines) $\rightarrow 0)$ is also possible. Therefore there is no need to do very fine discretization of the structure which saves memory and computation time. Fig. 5 gives the corresponding relative error of the computed values with increasing number of discretization lines with respect to HFSS solution.

Fig. 6 shows the dispersion curve for two fundamental modes of the same waveguide over normalized $d_{2}$. We have 


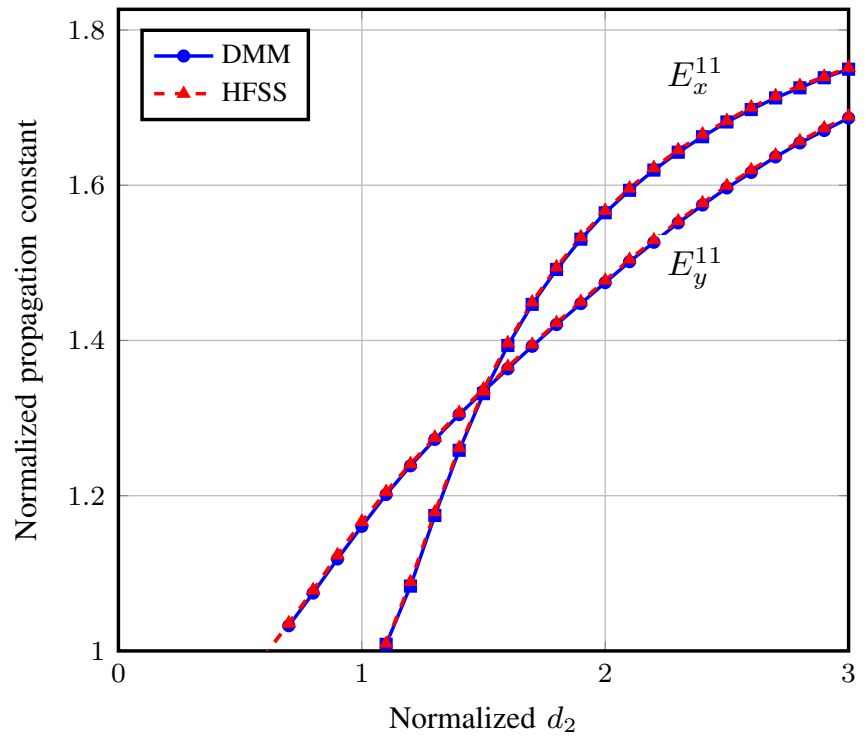

Fig. 6: Dispersion curve for insulated image waveguide.



Fig. 7: Relative error for two modes in insulated image waveguide with respect to HFSS solution (port mode calculation).

validated the results with the predicted results from ANSYS HFSS. The results also agree well with the results presented in [6] for MoL and finite difference technique. Fig. 7 gives the relative error for both the modes between DMM and HFSS results using only port mode calculation. Figs. 8 and 9 shows the normalized electric field distribution at the waveguide cross-section for $d_{2}=1$. We have used normal simulation in HFSS for field calculation. The figure shows that the field is concentrated mainly over the dielectric strip region and is maximum below the strip.

\section{B. Hybrid Waveguide}

To understand the coupling between the microstrip and the dielectric waveguide, we have examined the hybrid waveguide

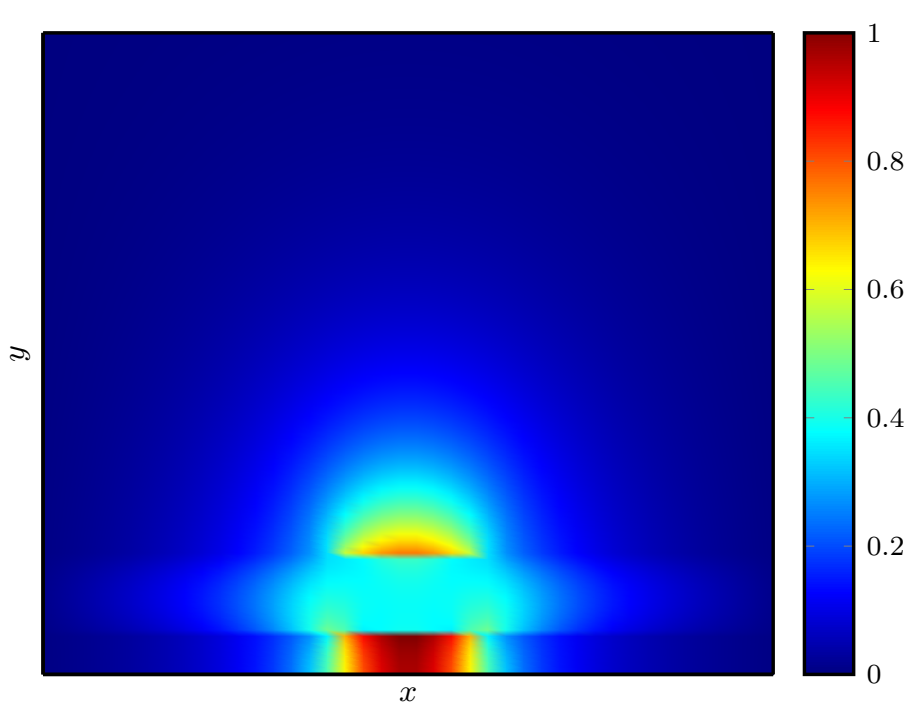

Fig. 8: Normalized field distribution on the cross-section of the insulated image waveguide using DMM.

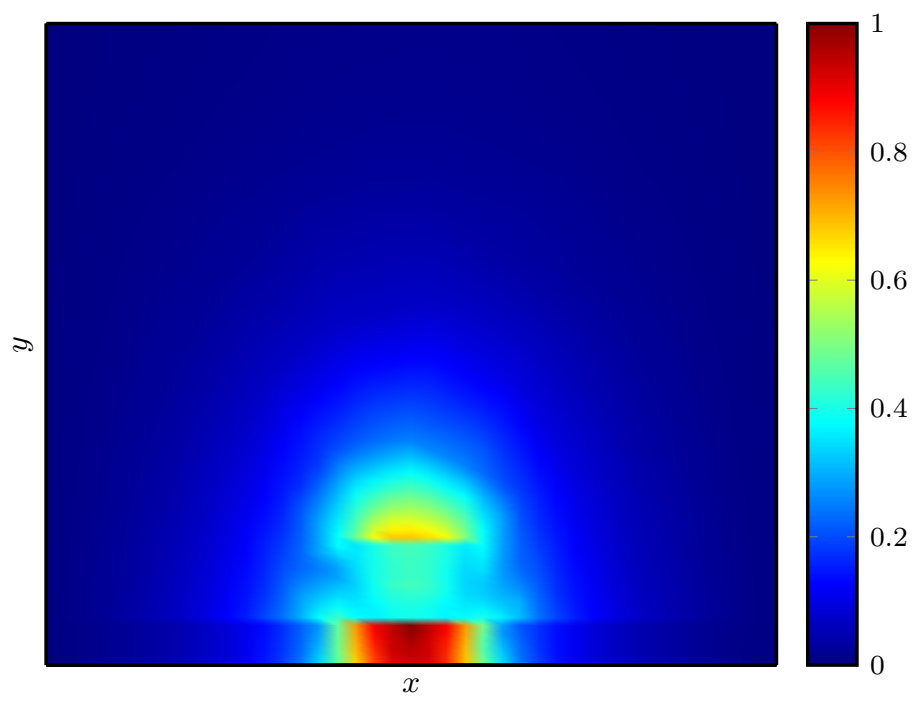

Fig. 9: Normalized field distribution on the cross-section of the insulated image waveguide using HFSS.

which consists of an inhomogeneous dielectric waveguide with microstrip, as shown in Fig. 10. The analysis gives the results for the coupled waveguide and microstrip (CG) when we move the dielectric slab from side wall to center. The results for the dielectric waveguide without microstrip (WG), and microstrip line without dielectric slab with $\overline{\bar{\varepsilon}}_{r 3}$ (MS) are also computed. We can divide the structure into four dielectric layers with three homogeneous layers and one inhomogeneous layer. The system matrix obtained for coupled hybrid waveguide is

$$
\left(\widetilde{\boldsymbol{Z}}_{e q_{d}}^{-1} \widetilde{\boldsymbol{V}}_{e q_{d}}+\widetilde{\boldsymbol{B}}_{e q_{u}} \widetilde{\boldsymbol{Z}}_{e q_{u}}^{-1}\right) \widetilde{\boldsymbol{E}}_{m}=\widetilde{\boldsymbol{J}}_{m}
$$

where

$$
\widetilde{\boldsymbol{K}}_{e q_{d}}=\widetilde{\boldsymbol{K}}_{1} \widetilde{\boldsymbol{K}}_{2} \quad \text { and } \quad \widetilde{\boldsymbol{K}}_{e q_{u}}=\widetilde{\boldsymbol{K}}_{3} \widetilde{\boldsymbol{K}}_{4}
$$

and $\widetilde{\boldsymbol{E}}_{m}$ and $\widetilde{\boldsymbol{J}}_{m}$ denote the electric field distribution and current density respectively over the interface with 


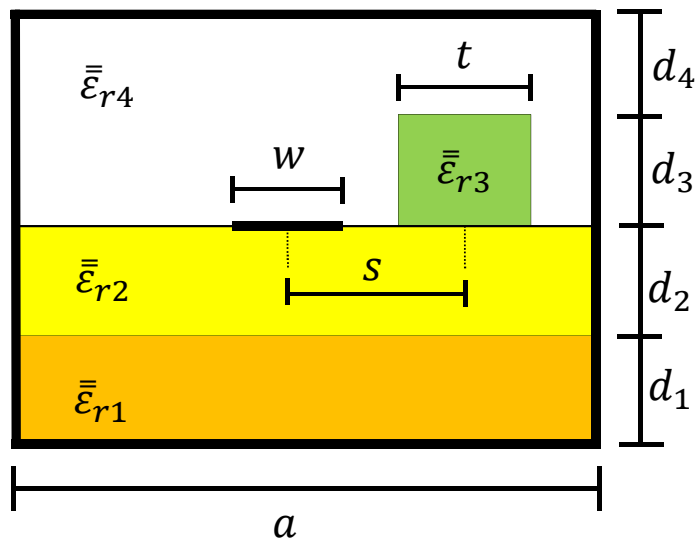

Fig. 10: Coupled microstrip and dielectric waveguide.

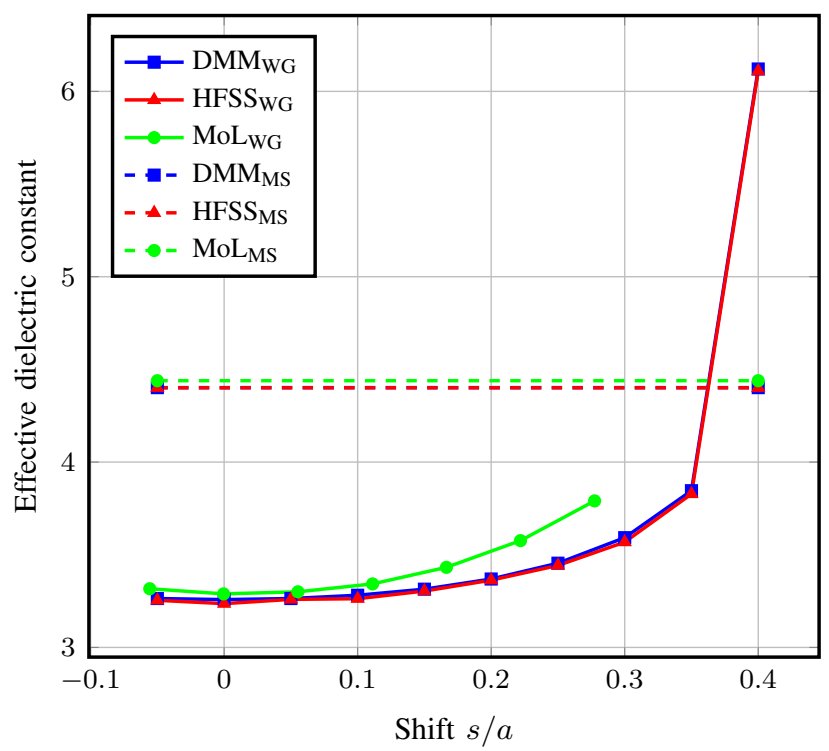

Fig. 11: Effective dielectric constant versus shift for isotropic waveguides (WG: dielectric waveguide only, MS: microstrip only).

metallization.

Figs. 11-14 show the effective dielectric constants with respect to the shift between microstrip and dielectric slab for isotropic and anisotropic dielectric waveguides. The data used for the analysis are $\varepsilon_{r 1}=1, \overline{\bar{\varepsilon}}_{r 2}=9.6$ or $(9.6,9,9.6)$, $\overline{\bar{\varepsilon}}_{r 3}=16$ or $(16,15,16), \varepsilon_{r 4}=1, a=7.112 \mathrm{~mm}, w=1.6$ $\mathrm{mm}, t=1.422 \mathrm{~mm}$ and $d_{1}=d_{2}=d_{3}=d_{4}=0.729 \mathrm{~mm}$. The computation is done at $30 \mathrm{GHz}$ frequency and with 5 $e$-lines on the strip. The DMM results agree well with the results obtained from the ANSYS HFSS for both isotropic and anisotropic medium. The isotropic results also agree with the results presented in [6].

We have done the programming of the code in MATLAB and performed the simulations on Intel i7-6600U CPU @ 2.6 $\mathrm{GHz}$ processor. The simulation for coupled waveguide and microstrip at $s / a=-0.05$ using DMM formulation took around 1 second and HFSS took 17 seconds with port mode calculation.

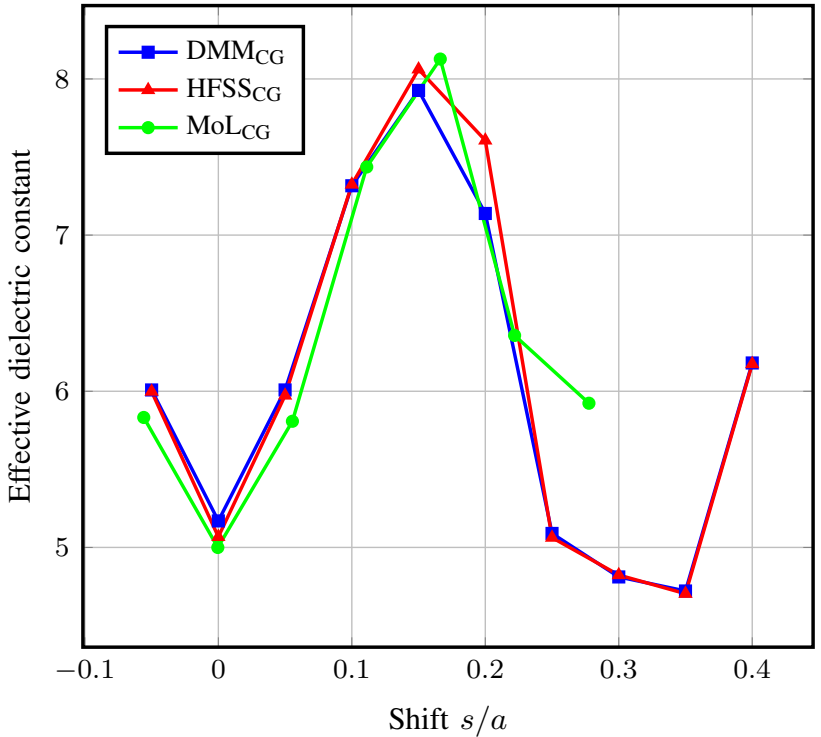

Fig. 12: Effective dielectric constant versus shift for isotropic coupled waveguide (CG: coupled guide).

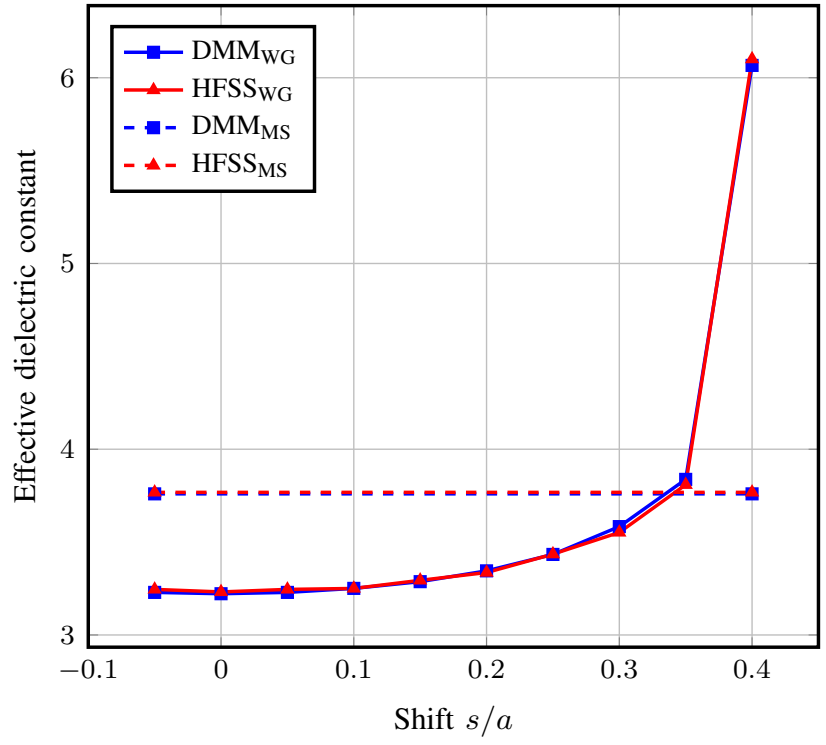

Fig. 13: Effective dielectric constant versus shift for anisotropic waveguides.

\section{Comparison Between Waveguides}

Next, we have done the comparison between the results obtained from different kinds of shielded waveguides. We have analyzed the conventional two-layer homogeneous dielectric waveguide, the quasi-planar waveguide with varying interface and the channel waveguide, as shown in Fig. 15. The quasi-planar interface of the waveguide has steep profile with the slope $>30^{\circ}$ at around $35 \%$ of the region.

We have considered only half of the structure for the analysis by placing a magnetic wall along the center due to symmetry. The material properties of the structure are $\bar{\varepsilon}_{r 1}=1.5$ or $(1.5,2,1), \varepsilon_{r 2}=1$ and $\mu_{r 1}=\mu_{r 2}=1$. The structure has width $a=4 d$ and height $b=2 d$. The width 


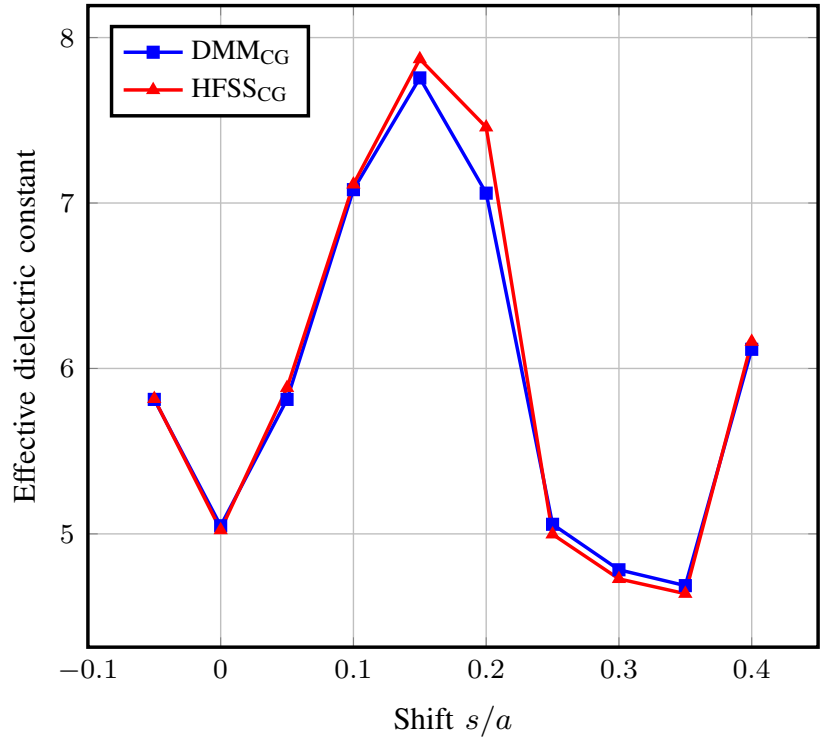

Fig. 14: Effective dielectric constant versus shift for anisotropic coupled waveguide.

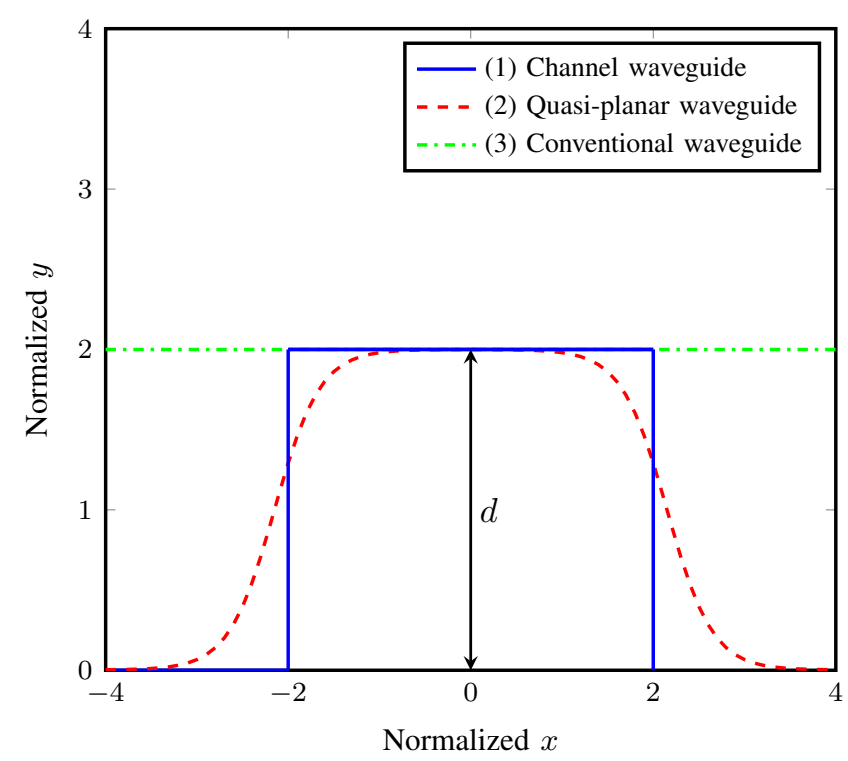

Fig. 15: Different analyzed waveguides (with $d=2$ ).

of the dielectric strip is taken to be $2 d$. For a quasi-planar waveguide, we can use any equation which can determine the shape of the interface. Here, we have used the function

$$
f(x)=0.001 b+(0.999 d /(1+\exp (B(x-A)))),
$$

with $A=\left(x_{1}+x_{2}\right) / 2, B=2 /\left|x_{1}-x_{2}\right|, x_{1}=0.95 b$ and $x_{2}=1.2 b$.

Figs. 16 and 17 give the normalized propagation constants computed from DMM with varying value of normalized $d$. The figures also compare the DMM results with the results obtained from HFSS. They show very good agreement between the results. It is clear from the figures that the results from the quasi-planar interface very well approximate the results from the channel structure.



Fig. 16: Dispersion curves for waveguides with isotropic medium. The different cases are shown in Fig. 15.



Fig. 17: Dispersion curves for waveguides with anisotropic medium.

\section{CONCLUSION}

The presented algorithm enables us to deal with different hybrid waveguides with inhomogeneous dielectric layers very accurately and efficiently. The formulation is simple and computationally efficient due to the semi-analytical approach. It uses straightforward circuit analysis technique and matrix multiplication to take into account several dielectric layers. Therefore, the multilayered structures and the graded-index waveguides can easily be analyzed. The DMM method of analysis has been demonstrated to be very efficient to analyze a variety of structures.

\section{REFERENCES}

[1] K. Wu and L. Han, "Hybrid integration technology of planar circuits and nrd-guide for cost-effective microwave and millimeter-wave 
applications," IEEE Trans. Microw. Theory Techn., vol. 45, no. 6, pp. 946-954, June 1997.

[2] Y. Cassivi, L. Perregrini, P. Arcioni, M. Bressan, K. Wu, and G. Conciauro, "Dispersion characteristics of substrate integrated rectangular waveguide," IEEE Microw. Wireless Compon. Lett., vol. 12, no. 9, pp. 333-335, Sept 2002.

[3] D. A. P. Bulla, B. . V. Borges, M. A. Romero, N. I. Morimoto, and L. G. Neto, "Design and fabrication of $\mathrm{SiO} /$ sub $2 / / \mathrm{Si} / \mathrm{sub} 3 / \mathrm{N} / \mathrm{sub} 4 /$ integrated-optics waveguides on silicon substrates," IEEE Trans. Microw. Theory Techn., vol. 50, no. 1, pp. 9-12, Jan 2002.

[4] R. Pashaie, "Fourier decomposition analysis of anisotropic inhomogeneous dielectric waveguide structures," IEEE Trans. Microw. Theory Techn., vol. 55, no. 8, pp. 1689-1696, Aug 2007.

[5] P. Berini and K. Wu, "Modeling lossy anisotropic dielectric waveguides with the method of lines," IEEE Trans. Microw. Theory Techn., vol. 44, no. 5, pp. 749-759, May 1996.

[6] R. Pregla, M. Koch, and W. Pascher, "Analysis of hybrid waveguide structures consisting of microstrips and dielectric waveguides," in 1987 17th European Microwave Conference, Sep. 1987, pp. 927-932.

[7] Y. Lu and F. A. Fernandez, "An efficient finite element solution of inhomogeneous anisotropic and lossy dielectric waveguides," IEEE Trans. Microw. Theory Techn., vol. 41, no. 6, pp. 1215-1223, June 1993.

[8] S. Akiba and H. A. Haus, "Variational analysis of optical waveguides with rectangular cross section,” Appl. Opt., vol. 21, no. 5, pp. 804-808, Mar 1982

[9] N. Eti and H. Kurt, "Model analysis of ridge and rib types of silicon waveguides with void compositions," IEEE J. Quantum Electron., vol. 52, no. 10, pp. 1-7, Oct 2016.

[10] A. Dreher and T. Rother, "New aspects of the method of lines," IEEE Microw. Guided Wave Lett., vol. 5, no. 11, pp. 408-410, Nov 1995.

[11] V. Kamra and A. Dreher, "Full-wave equivalent circuit for the analysis of multilayered microwave structures with anisotropic layers," Electronics Letters, vol. 54, no. 3, pp. 153-155, 2018.

[12] A. Dreher and A. Ioffe, "Analysis of microstrip lines in multilayer structures of arbitrarily varying thickness," IEEE Microwave and Guided Wave Letters, vol. 10, no. 2, pp. 52-54, Feb 2000.

[13] V. Kamra and A. Dreher, "Efficient analysis of multiple microstrip transmission lines with anisotropic substrates," IEEE Microw. Wireless Compon. Lett., vol. 28, no. 8, pp. 636-638, Aug 2018.

[14] V. Kamra and A. Dreher, "Multilayered transmission lines on quasi-planar substrates with anisotropic medium," Advances in Radio Science, vol. 17, pp. 77-82, Sep. 2019.

[15] M. V. T. Heckler and A. Dreher, "Analysis of cylindrical microstrip lines using the discrete mode matching method," IEEE Microwave and Wireless Components Letters, vol. 16, no. 7, pp. 392-394, July 2006.

[16] V. Kamra and A. Dreher, "Analysis of circular and noncircular waveguides and striplines with multilayered uniaxial anisotropic medium," IEEE Trans. Microw. Theory Techn., vol. 67, no. 2, pp. 584-591, Feb 2019.

[17] _ - "Analysis of anisotropic inhomogeneous dielectric waveguides with discrete mode matching method," in International Microwave Symposium, June 2019.



Veenu Kamra was born in Bikaner, India, in 1990. She received Bachelor of Technology degree in Electronics \& Communication Engineering in 2012 from the Rajasthan Technical University, India, and Master of Technology Degree in Electronics \& Communication Engineering (specialization in RF and Microwaves) in 2014 from the Indian Institute of Technology Roorkee (IITR), India.

From August 2014 to January 2016, she worked as an Assistant Professor in Jaipur Engineering College \& Research Centre, India. Since February 2016, she has been working towards her $\mathrm{PhD}$ with the Antenna Group, Institute of Communications and Navigation, German Aerospace Center (DLR). Her current research interest is numerical techniques for microwave structures.

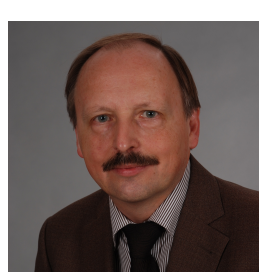

Achim Dreher (M'92-SM'99) received the Dipl.-Ing. (M.S.) degree from the Technische Universität Braunschweig, Braunschweig, Germany, in 1983, and the Dr.-Ing. (Ph.D.) degree from the FernUniversität, Hagen, Germany, in 1992, both in electrical engineering.

From 1983 to 1985 , he was a Development Engineer with Rohde \& Schwarz GmbH \& Co. KG, München, Germany. From 1985 to 1992 he was a Research Assistant, and from 1992 to 1997, he was a Senior Research Engineer with the Department of Electrical Engineering, FernUniversität. Since 1997 he has been with the Institute of Communications and Navigation, German Aerospace Center (DLR), Oberpfaffenhofen, Germany, where he is currently Head of the Antenna Research Group. His current research interests include analytical and numerical techniques for conformal antennas and microwave structures, smart antennas for satellite communications and navigation, and antenna technology for radar applications.

Dr. Dreher is a member of the VDE/ITG expert committee on antennas and serves as scientific coordinator and permanent lecturer for the Carl Cranz Series for Scientific Education in the area of smart antenna systems. 